\title{
Sharp quantitative nonembeddability of the Heisenberg group into superreflexive Banach spaces
}

\author{
Tim Austin ${ }^{1}$, Assaf Naor ${ }^{2}$ and Romain Tessera
}

Abstract. Let $\mathbb{H}$ denote the discrete Heisenberg group, equipped with a word metric $d_{W}$ associated to some finite symmetric generating set. We show that if $(X,\|\cdot\|)$ is a $p$-convex Banach space then for any Lipschitz function $f: \mathbb{H} \rightarrow X$ there exist $x, y \in \mathbb{H}$ with $d_{W}(x, y)$ arbitrarily large and

$$
\frac{\|f(x)-f(y)\|}{d_{W}(x, y)} \lesssim\left(\frac{\log \log d_{W}(x, y)}{\log d_{W}(x, y)}\right)^{1 / p}
$$

We also show that any embedding into $X$ of a ball of radius $R \geq 4$ in $\mathbb{H}$ incurs bi-Lipschitz distortion that grows at least as a constant multiple of

$$
\left(\frac{\log R}{\log \log R}\right)^{1 / p} .
$$

Both (1) and (2) are sharp up to the iterated logarithm terms. When $X$ is Hilbert space we obtain a representation-theoretic proof yielding bounds corresponding to (1) and (2) which are sharp up to a universal constant.

Mathematics Subject Classification (2010). 46B85, 30L05, 20F65.

Keywords. Bi-Lipschitz embedding, Heisenberg group, superreflexive Banach spaces.

\section{Introduction}

Let $\mathbb{H} \stackrel{\text { def }}{=}\langle a, b| a b a^{-1} b^{-1}$ is central $\rangle$ denote the discrete Heisenberg group, with canonical generators $a, b \in \mathbb{H}$. We let $d_{W}(\cdot, \cdot)$ denote the left-invariant word metric on $\mathbb{H}$ associated to the symmetric generating set $S \stackrel{\text { def }}{=}\left\{a, b, a^{-1}, b^{-1}\right\}$.

A Banach space $\left(X,\|\cdot\|_{X}\right)$ is superreflexive if it admits an equivalent uniformly convex norm, i.e., a norm $\|\cdot\|$ satisfying $\alpha\|x\|_{X} \leq\|x\| \leq \beta\|x\|_{X}$ for some $\alpha, \beta>0$

\footnotetext{
${ }^{1}$ First author partially supported by a fellowship from Microsoft Corporation.

${ }^{2}$ Second author supported by NSF grants CCF-0635078 and CCF-0832795, BSF grant 2006009, and the Packard Foundation.
} 
and all $x \in X$, such that for all $\varepsilon \in(0,1)$ there exists $\delta>0$ for which we have

$$
\|x\|=\|y\|=1 \wedge\|x-y\|=\varepsilon \Longrightarrow\|x+y\| \leq 2-\delta .
$$

Here we prove the following result:

Theorem 1.1. Let $\left(X,\|\cdot\|_{X}\right)$ be a superreflexive Banach space. Then there exist $c, C>0$ such that for every $f: \mathbb{H} \rightarrow X$ which is 1-Lipschitz with respect to the metric $d_{W}$, there are $x, y \in X$ with $d_{W}(x, y)$ arbitrarily large and

$$
\frac{\|f(x)-f(y)\|_{X}}{d_{W}(x, y)} \leq \frac{C}{\left(\log d_{W}(x, y)\right)^{c}} .
$$

The fact that $\mathbb{H}$ does not admit a bi-Lipschitz embedding into any superreflexive Banach space was proved in [18], [6]. These proofs use an argument of Semmes [25], based on a natural extension of Pansu's differentiability theorem [22].

A natural way to quantify the extent to which $\mathbb{H}$ does not admit a bi-Lipschitz embedding into $\left(X,\|\cdot\|_{X}\right)$ is via Gromov's notion [13], Section 7.3, of compression rate, defined for a Lipschitz function $f: \mathbb{H} \rightarrow X$ as the largest function $\omega_{f}:(0, \infty) \rightarrow$ $[0, \infty)$ such that for all $x, y \in \mathbb{H}$ we have $\|f(x)-f(y)\|_{X} \geq \omega_{f}\left(d_{W}(x, y)\right)$. The fact that $\mathbb{H}$ does not admit a bi-Lipschitz embedding into a superreflexive Banach space $X$ means that $\liminf _{t \rightarrow \infty} \omega_{f}(t) / t=0$ for all Lipschitz functions $f: \mathbb{H} \rightarrow X$. The differentiability-based proof of this nonembeddability result involves a limiting argument that does not give information on the rate at which $\omega_{f}(t) / t$ vanishes. Theorem 1.1 supplies such information, via an approach which is different from the arguments in [18], [6].

Cheeger and Kleiner proved [7] that $\mathbb{H}$ does not admit a bi-Lipschitz embedding into $L_{1}$. In [8] it was shown that there exists $c>0$ such that for any Lipschitz function $f: \mathbb{H} \rightarrow L_{1}$ we have $\omega_{f}(t) / t \leq 1 /(\log t)^{c}$ for arbitrarily large $t$. This result covers Theorem 1.1 when the superreflexive Banach space $X$ admits a biLipschitz embedding into $L_{1}$ : such spaces include $L_{p}$ for $p \in(1,2]$. Theorem 1.1 is new even for spaces such as $L_{p}$ for $p \in(2, \infty)$, which do not admit a bi-Lipschitz embedding into $L_{1}$ (see [3]). Moreover, our method yields sharp results, while the constant $c$ obtained in [8] is far from sharp.

In order to state our sharp version of Theorem 1.1, we recall the following important theorem of Pisier [23] ([Theorem 3.1]): if $X$ is superreflexive then it admits an equivalent norm $\|\cdot\|$ for which there exist $p \geq 2$ and $K>0$ satisfying the following improvement of (3):

$$
\left\|\frac{x+y}{2}\right\|^{p} \leq \frac{\|x\|^{p}+\|y\|^{p}}{2}-\frac{1}{K^{p}}\left\|\frac{x-y}{2}\right\|^{p} \quad \text { for all } x, y \in X .
$$

A Banach space admitting an equivalent norm satisfying (4) is said to be $p$-convex. If $(X,\|\cdot\|)$ satisfies (4) then the infimum over those $K>0$ satisfying (4) is denoted 
$K_{p}(X)$. For concreteness, when $p \in(1,2]$ we have $K_{2}\left(L_{p}\right) \leq 1 / \sqrt{p-1}$ and for $p \geq 2$ we have $K_{p}\left(L_{p}\right) \leq 1$ (see [2]).

The following theorem is a refinement of Theorem 1.1.

Theorem 1.2. Assume that the Banach space $(X,\|\cdot\|)$ satisfies (4). Let $f: \mathbb{H} \rightarrow X$ be a 1-Lipschitz function. Then for every $t \geq 3$ there exists an integer $t \leq n \leq t^{2}$ such that ${ }^{1}$

$$
\frac{\omega_{f}(n)}{n} \lesssim K_{p}(X)\left(\frac{\log \log n}{\log n}\right)^{1 / p}
$$

The estimate (5) is sharp up to the iterated logarithm term. Indeed, $L_{p}$ is $p$-convex when $p \in[2, \infty)$, and in [27], [26] it was shown that there exists $f: \mathbb{H} \rightarrow L_{p}$ satisfying

$$
\frac{\omega_{f}(n)}{n} \gtrsim \frac{1}{(\log n)^{1 / p} \log \log n}
$$

for all $n \geq 3$ (we refer to [26] for a more refined result of this type).

Our proof of Theorem 1.2 circumvents the difficulties involved with proving quantitative variants of differentiability results by avoiding the need to reason about arbitrary Lipschitz mappings. Instead, we start by using a simple result from [21] which reduces the problem to equivariant mappings. Specifically, in [21], Theorem 9.1, it is shown that if $X$ satisfies (4) and $f: \mathbb{H} \rightarrow X$ is 1-Lipschitz, then there exists a Banach space $Y$ that also satisfies (4), with $K_{p}(Y)=K_{p}(X)$ (in fact, $Y$ is finitely representable in $\ell_{p}(X)$ ), an action $\pi$ of $\mathbb{H}$ on $Y$ by linear isometric automorphisms, and a 1-cocycle $F: \mathbb{H} \rightarrow Y$ (i.e., $F(x y)=\pi(x) F(y)+F(x)$ for all $x, y \in \mathbb{H}$ ) with $\omega_{F}=\omega_{f}$. Thus, in proving Theorem 1.2 it suffices to assume that $f$ itself is a 1-cocycle. We note that if $X$ is Hilbert space then $Y$ is also Hilbert space; this is an older result of Gromov (see [10]). More generally, when $X=L_{p}$ then it is shown in [21] that we can take $Y=L_{p}$.

Having reduced the problem to 1-cocycles, our starting point is a (non-quantitative) proof, explained in Section 2, showing that if $X$ is an ergodic Banach space, then for every 1-cocycle $f: \mathbb{H} \rightarrow X$ we have $\liminf _{t \rightarrow \infty} \omega_{f}(t) / t=0$. It turns out that the ideas of this proof, which crucially use the fact that $f$ is a 1-cocycle, can be (nontrivially) adapted to yield Theorem 1.2.

Recall that $X$ is ergodic if for every linear isometry $T: X \rightarrow X$ and every $x \in X$ the sequence $\left\{\frac{1}{n} \sum_{j=0}^{n-1} T^{j} x\right\}_{n=1}^{\infty}$ converges in norm. Reflexive spaces, and hence also superreflexive spaces, are ergodic (see [11], p. 662). If a Banach space $X$ has the property that all Banach spaces that are finitely representable in $X$ are ergodic, then $X$ must be superreflexive [4]. Thus, when using the reduction to 1-cocyles based on the result of [21], the class of Banach spaces to which it naturally applies is the class of superreflexive spaces.

\footnotetext{
${ }^{1}$ In (5), and in the rest of this paper, the notation $\lesssim, \gtrsim$ denotes the corresponding inequalities up to a universal multiplicative factor. The notation $A \asymp B$ stands for $A \lesssim B \wedge B \lesssim A$.
} 
1.1. Bi-Lipschitz distortion of balls. For $R \geq 1$ let $B_{R}=\left\{x \in \mathbb{H}: d_{W}(e, x) \leq\right.$ $R$ \} denote the ball of radius $R$ centered at the identity element $e \in \mathbb{H}$. The biLipschitz distortion of $\left(B_{R}, d_{W}\right)$ in $(X,\|\cdot\|)$, denoted $c_{X}\left(B_{R}\right)$, is the infimum over those $D \geq 1$ such that there exists $f: B_{R} \rightarrow X$ satisfying

$$
d_{W}(x, y) \leq\|f(x)-f(y)\| \leq D d_{W}(x, y) \text { for all } x, y \in B_{R} .
$$

Another way to measure the extent to which $\mathbb{H}$ does not admit a bi-Lipschitz embedding into $X$ is via the rate at which $c_{X}\left(B_{R}\right)$ grows to $\infty$ with $R$. In [8] it was shown that

$$
c_{L_{1}}\left(B_{R}\right) \gtrsim(\log R)^{c}
$$

for some universal constant $c>0$. This result is of importance due to an application to theoretical computer science; see [9], [8], [20] for a detailed discussion. Evaluating the supremum over those $c>0$ satisfying (7) remains an important open problem. Theorem 1.2 implies the following sharp bound on the bi-Lipschitz distortion of $B_{R}$ into a $p$-convex Banach space:

Theorem 1.3. If a Banach space $(X,\|\cdot\|)$ satisfies (4) then for every $R \geq 4$ we have

$$
c_{X}\left(B_{R}\right) \gtrsim \frac{1}{K_{p}(X)}\left(\frac{\log R}{\log \log R}\right)^{1 / p} .
$$

Thus in particular for $p \in(1,2]$ we have $c_{L_{p}}\left(B_{R}\right) \gtrsim \sqrt{p-1} \cdot(\log R)^{\frac{1}{2}-o(1)}$ and for $p \geq 2$ we have $c_{L_{p}}\left(B_{R}\right) \gtrsim(\log R)^{\frac{1}{p}-o(1)}$. Theorem 1.3 is a formal consequence of Theorem 1.2. The simple deduction of Theorem 1.3 from Theorem 1.2 is presented in Section 6. It follows from the results of [1], [24] (see the explanation in [15], [26]) that for every $p \geq 2$ we have $c_{L_{p}}\left(B_{R}\right) \lesssim(\log R)^{1 / p}$. Thus Theorem 1.3 is sharp up to iterated logarithms.

1.2. The case of Hilbert space. In Section 7 we prove the following Poincaré-type inequality for functions on $\mathbb{H}$ taking value in Hilbert space:

Theorem 1.4. For every $f: \mathbb{H} \rightarrow L_{2}$ and every $R \in \mathbb{N}$ we have

$$
\sum_{x \in B_{R}} \sum_{k=1}^{R^{2}} \frac{\left\|f\left(x c^{k}\right)-f(x)\right\|_{2}^{2}}{k^{2}} \lesssim \sum_{x \in B_{22}}\left(\|f(x a)-f(x)\|_{2}^{2}+\|f(x b)-f(x)\|_{2}^{2}\right) .
$$

This result has the following two sharp consequences. First, assume that $\theta:(0, \infty) \rightarrow[0, \infty)$ is nondecreasing, and that $\theta \leq \omega_{f}$ for some 1-Lipschitz $f: \mathbb{H} \rightarrow L_{2}$. Then since $\left|B_{22 R}\right| \asymp\left|B_{R}\right|$ and $d_{W}\left(c^{k}, e\right) \asymp \sqrt{k}$ for all $k \in \mathbb{N}$, 
inequality (8) implies that

$$
\frac{1}{2} \int_{1}^{\infty} \frac{\theta(t)^{2}}{t^{3}} d t=\int_{1}^{\infty} \frac{\theta(\sqrt{s})^{2}}{s^{2}} d s \lesssim \sum_{k=1}^{\infty} \frac{\theta(\sqrt{k})^{2}}{k^{2}} \lesssim 1 .
$$

Combined with Theorem 1 of [27], we obtain

Corollary 1.5. A nondecreasing function $\theta:(0, \infty) \rightarrow[0, \infty)$ satisfies $\theta \leq \omega_{f}$ for some Lipschitz function $f: \mathbb{H} \rightarrow L_{2}$ if and only if

$$
\int_{1}^{\infty}\left(\frac{\theta(t)}{t}\right)^{2} \frac{d t}{t}<\infty .
$$

A second corollary of Theorem 1.4 yields a sharp bound (up to universal constants) on $c_{L_{2}}\left(B_{R}\right)$. Indeed, fix $R \geq 2$ and assume that $f: B_{R} \rightarrow L_{2}$ satisfies $d_{W}(x, y) \leq$ $\|f(x)-f(y)\| \leq D d_{W}(x, y)$ for all $x, y \in B_{R}$. Let $f^{*}: \mathbb{H} \rightarrow L_{2}$ have Lipschitz constant at most $2 D$ and coincide with $f$ on $B_{R / 2}$ (see equation (41) for an explicit formula defining such an extension $f^{*}$ ). It follows from (8) applied to $f^{*}$ that $D^{2} \gtrsim \sum_{k=1}^{R} \frac{1}{k} \gtrsim \log R$. Thus $c_{L_{2}}\left(B_{R}\right) \gtrsim \sqrt{\log R}$. In conjunction with the previously quoted upper bound on $c_{L_{2}}\left(B_{R}\right)$, we have

Corollary 1.6. For every $R \geq 2$ we have $c_{L_{2}}\left(B_{R}\right) \asymp \sqrt{\log R}$.

Roughly speaking, the proof of Theorem 1.4 proceeds via a reduction to the case of 1-cocycles corresponding to the irreducible representations of $\mathbb{H}$ (see Section 7). But actually, since the representation theory of the continuous Heisenberg group is simpler than the representation theory of the discrete Heisenberg group $\mathbb{H}$, we first apply a discretization argument which reduces Theorem 1.4 to an inequality on the real Heisenberg group. Then an averaging argument reduces the proof to an inequality on cocycles. Every unitary representation of the continuous Heisenberg group decomposes as a direct integral of irreducibles, and cocycles themselves can be decomposed accordingly. Since the desired inequality involves a sum of squares of norms, it suffices to prove it for cocycles corresponding to irreducible representations (that is, for each direct integrand separately). The computation for irreducible representations is carried out in Section 7.1.

\section{Sublinear growth of Heisenberg cocycles in ergodic spaces}

Write $c \stackrel{\text { def }}{=}[a, b]=a b a^{-1} b^{-1}$. Thus $c$ lies in the center of $\mathbb{H}$ and for every $n \in \mathbb{N}$ we have $d_{W}\left(c^{n^{2}}, e_{\mathbb{H}}\right)=4 n$ (in fact $\left.c^{n^{2}}=\left[a^{n}, b^{n}\right]=a^{n} b^{n} a^{-n} b^{-n}\right)$.

Let $(X,\|\cdot\|)$ be a Banach space and $\pi: G \rightarrow \operatorname{Aut}(X)$ be an action of $\mathbb{H}$ on $X$ by linear isometric automorphisms. In addition let $f \in Z^{1}(\pi)$ be a 1-cocycle, so 
$f: \mathbb{H} \rightarrow X$ and for all $x, y \in \mathbb{H}$ we have $f(x y)=\pi(x) f(y)+f(x)$. We assume in what follows that $f$ is 1-Lipschitz, or equivalently that $\max \{\|f(a)\|,\|f(b)\|\}=1$.

In this section we quickly show that if $X$ is an ergodic Banach space then $\liminf _{t \rightarrow \infty} \omega_{f}(t) / t=0$, but without obtaining any quantitative bounds.

If $X$ is ergodic then the operator on $X$ defined by

$$
P x \stackrel{\text { def }}{=} \lim _{N \rightarrow \infty} \frac{1}{N} \sum_{n=0}^{N-1} \pi(c)^{n} x
$$

is a contraction onto the subspace $X_{0} \subseteq X$ of $\pi(c)$-invariant vectors, and since $P x=x$ for any $x \in X_{0}$ it follows at once that $P$ is idempotent. Also, since $c$ is central in $\mathbb{H}$, the projection $P$ commutes with $\pi(g)$ for all $g \in \mathbb{H}$, and hence the maps $g \mapsto P f(g)$ and $g \mapsto(I-P) f(g)$ are both still members of $Z^{1}(\pi)$. Since $P$ and $I-P$ are bounded, these cocycles are both still Lipschitz functions from $\mathbb{H}$ to $X$.

We complete the proof by showing that for any $\varepsilon>0$ we have

$$
\left\|f\left(c^{N^{2}}\right)\right\| \leq 2 \varepsilon d_{W}\left(e_{\mathbb{H}}, c^{N^{2}}\right) \leq 8 \varepsilon N
$$

for all sufficiently large $N$. To prove this we consider the two cocycles $P f$ and $(I-P) f$ separately. On the one hand, $P f$ takes values among the $\pi(c)$-invariant vectors, and hence the cocycle identity implies that

$$
P f\left(c^{N}\right)=\sum_{n=0}^{N-1} \pi(c)^{n} \operatorname{Pf}(c)=N P f(c),
$$

and therefore $\left\|P f\left(c^{N}\right)\right\|=N\|P f(c)\|$. However, $\left\|P f\left(c^{N}\right)\right\| \leq d_{W}\left(e_{\mathbb{H}}, c^{N}\right) \lesssim$ $\sqrt{N}$, so these relations are compatible only if $P f(c)=0$.

On the other hand, let $\tilde{f} \stackrel{\text { def }}{=}(I-P) f$ and for each $K \geq 1$,

$$
v_{K} \stackrel{\text { def }}{=}-\frac{1}{K} \sum_{k=1}^{K} f\left(c^{k}\right) \text {. }
$$

Observe from the cocycle identity and the centrality of $c$ that

$$
\begin{aligned}
-\pi(g) v_{K}+\tilde{f}(g) & =\frac{1}{K} \sum_{k=1}^{K}\left(\pi(g) \tilde{f}\left(c^{k}\right)+\tilde{f}(g)\right)=\frac{1}{K} \sum_{k=1}^{K} \tilde{f}\left(g c^{k}\right) \\
& =\frac{1}{K} \sum_{k=1}^{K} \tilde{f}\left(c^{k} g\right)=\frac{1}{K} \sum_{k=1}^{K} \pi\left(c^{k}\right) \tilde{f}(g)-v_{K} .
\end{aligned}
$$

Re-arranging (12) gives

$$
\tilde{f}(g)=\pi(g) v_{K}-v_{K}+\frac{1}{K} \sum_{k=1}^{K} \pi(c)^{k} \tilde{f}(g) .
$$


For any fixed $g \in \mathbb{H}$ the last term of this right-hand side of (13) converges to $P(I-P) f(g)=0$ (using again that $X$ is ergodic), and so in particular once $K$ is sufficiently large we obtain that for all $g \in \mathbb{H}$ we have,

$$
\max \left\{\left\|\tilde{f}\left(a^{ \pm 1}\right)-\left(\pi\left(a^{ \pm 1}\right) v_{K}-v_{K}\right)\right\|,\left\|\tilde{f}\left(b^{ \pm 1}\right)-\left(\pi\left(b^{ \pm 1}\right) v_{K}-v_{K}\right)\right\|\right\} \leq \varepsilon .
$$

Having obtained this approximation to $\tilde{f}$ by a coboundary, let $c^{N^{2}}=s_{1} s_{2} \cdots s_{4 N}$ be an expression for $c^{N^{2}}$ as a word in $S$, and observe from another appeal to the cocycle identity that

$$
\begin{aligned}
\tilde{f}\left(c^{N^{2}}\right) & =\sum_{i=0}^{4 N-1} \pi\left(s_{1} s_{2} \cdots s_{i}\right) \tilde{f}\left(s_{i+1}\right) \\
& =\sum_{i=0}^{4 N-1} \pi\left(s_{1} s_{2} \cdots s_{i}\right)\left(\pi\left(s_{i+1}\right) v_{K}-v_{K}\right)+R_{N} \\
& =\pi\left(s_{1} s_{2} \cdots s_{4 N}\right) v_{K}-v_{K}+R_{N}
\end{aligned}
$$

for some remainder $R_{N}$ which is a sum of $4 N$ terms all of norm at most $\varepsilon$. Since the action $\pi$ is isometric and we may let $N$ grow independently of $K$ we obtain

$$
\left\|\tilde{f}\left(c^{N^{2}}\right)\right\| \leq 2\left\|v_{K}\right\|+\left\|R_{N}\right\| \leq 8 \varepsilon N
$$

for all sufficiently large $N$. Since $\varepsilon$ was arbitrary and $\tilde{f}\left(c^{N^{2}}\right)=f\left(c^{N^{2}}\right)$ by our analysis of $P f$ above, this completes the proof of (11).

\section{A uniform convexity lemma for ergodic averages}

We prove here a simple lemma on the behavior of ergodic averages in $p$-convex Banach spaces.

Lemma 3.1. Assume that $(X,\|\cdot\|)$ satisfies (4). Fix $z \in X$ and an operator $T: X \rightarrow$ $X$ with $\|T\| \leq 1$. For every integer $n \geq 0$ write

$$
s_{n} \stackrel{\text { def }}{=} \frac{1}{2^{n}} \sum_{j=0}^{2^{n}-1} T^{j} z .
$$

Then for every $\ell \in \mathbb{N}$ we have

$$
\sum_{i=0}^{\infty} \frac{1}{2^{\ell}} \sum_{j=0}^{2^{\ell}-1}\left\|s_{(i+1) \ell}-T^{j 2^{i \ell}} s_{i \ell}\right\|^{p} \leq(2 K)^{p}\|z\|^{p} .
$$


Proof. A consequence of (4) is that for every $x_{1}, \ldots, x_{n} \in X$ we have

$$
\frac{1}{n} \sum_{i=1}^{n}\left\|x_{i}-\frac{1}{n} \sum_{j=1}^{n} x_{j}\right\|^{p} \leq(2 K)^{p}\left(\frac{1}{n} \sum_{i=1}^{n}\left\|x_{i}\right\|^{p}-\left\|\frac{1}{n} \sum_{i=1}^{n} x_{i}\right\|^{p}\right) .
$$

For the derivation of (15) from (4) see [19], Lemma 3.1.

Due to the identity

$$
s_{(i+1) \ell}=\frac{1}{2^{\ell}} \sum_{j=0}^{2^{\ell}-1} T^{j 2^{i \ell}} s_{i \ell},
$$

inequality (15) implies that

$$
\begin{aligned}
\frac{1}{2^{\ell}} \sum_{j=0}^{2^{\ell}-1}\left\|T^{j 2^{i \ell}} s_{i \ell}-s_{(i+1) \ell}\right\|^{p} & \leq(2 K)^{p}\left(\frac{1}{2^{\ell}} \sum_{j=0}^{2^{\ell}-1}\left\|T^{j 2^{i \ell}} s_{i \ell}\right\|^{p}-\left\|s_{(i+1) \ell}\right\|^{p}\right) \\
& \leq(2 K)^{p}\left(\left\|s_{i \ell}\right\|^{p}-\left\|s_{(i+1) \ell}\right\|^{p}\right) .
\end{aligned}
$$

The desired inequality (14) now follows by summing (16) over $i \in\{0,1, \ldots\}$.

\section{Estimates for Heisenberg cocycles}

Let $\pi: \mathbb{H} \rightarrow \operatorname{Aut}(X)$ and $f \in Z^{1}(\pi)$ be as in Section 2. For every $n \in \mathbb{N}$ define a linear operator $P_{n}: X \rightarrow X$ by

$$
P_{n} \stackrel{\text { def }}{=} \frac{1}{2^{n}} \sum_{j=0}^{2^{n}-1} \pi(c)^{j}
$$

Thus $\left\|P_{n}\right\| \leq 1$.

Lemma 4.1. Assume that $(X,\|\cdot\|)$ satisfies (4). Then for every $\ell, k, m \in \mathbb{N}$ there exist integers $i \in[k+1, k+m]$ and $j \in\left[0,2^{\ell}-1\right]$ satisfying for all $n \in \mathbb{N}$,

$$
\left\|\pi\left(c^{-j 2^{i \ell}}\right) P_{(i+1) \ell} f\left(c^{n^{2}}\right)-P_{i \ell} f\left(c^{n^{2}}\right)\right\| \leq \frac{16 K n}{m^{1 / p}} .
$$

Proof. Consider the Banach space $Y=X \oplus X$, equipped with the norm

$$
\|(x, y)\|_{Y}=\left(\|x\|^{p}+\|y\|^{p}\right)^{1 / p} .
$$

We also define $T: Y \rightarrow Y$ by $T(x, y)=(\pi(c) x, \pi(c) y)$. Then $\|T\| \leq 1$. Since $\left(Y,\|\cdot\|_{Y}\right)$ satisfies (4) we may apply Lemma 3.1 to $z=(f(a), f(b)) \in Y$, obtaining 
the following estimate:

$$
\begin{gathered}
(4 K)^{p} \geq \sum_{i=k+1}^{k+m} \frac{1}{2^{\ell}} \sum_{j=0}^{2^{\ell}-1}\left(\left\|P_{(i+1) \ell} f(a)-\pi\left(c^{j 2^{i \ell}}\right) P_{i \ell} f(a)\right\|^{p}\right. \\
\left.+\left\|P_{(i+1) \ell} f(b)-\pi\left(c^{j 2^{i \ell}}\right) P_{i \ell} f(b)\right\|^{p}\right) \\
=\sum_{i=k+1}^{k+m} \frac{1}{2^{\ell}} \sum_{j=0}^{2^{\ell}-1}\left(\left\|\pi\left(c^{-j 2^{i \ell}}\right) P_{(i+1) \ell} f(a)-P_{i \ell} f(a)\right\|^{p}\right. \\
\geq m \min _{\substack{k+1 \leq i \leq k+m \\
0 \leq j \leq 2^{\ell}-1}}\left(\left\|\pi\left(c^{-j 2^{i \ell}}\right) P_{(i+1) \ell} f(a)-P_{i \ell} f(a)\right\|^{p}\right. \\
\left.+\left\|\pi\left(c^{-j 2^{i \ell}}\right) P_{(i+1) \ell} f(b)-P_{i \ell} f(b)\right\|^{p}\right) .
\end{gathered}
$$

It follows that there exist integers $i \in[k+1, k+m], j \in\left[0,2^{\ell}-1\right]$ such that

$$
\begin{aligned}
& \max \left\{\left\|\pi\left(c^{-j 2^{i \ell}}\right) P_{(i+1) \ell} f(a)-P_{i \ell} f(a)\right\|,\right. \\
& \left.\left\|\pi\left(c^{-j 2^{i \ell}}\right) P_{(i+1) \ell} f(b)-P_{i \ell} f(b)\right\|\right\} \leq \frac{4 K}{m^{1 / p}} .
\end{aligned}
$$

Consider the operator

$$
Q_{n} \stackrel{\text { def }}{=} \frac{1}{n} \sum_{i=0}^{n-1} \pi(a)^{i}
$$

The cocycle identity implies that $f\left(a^{n}\right)=n Q_{n} f(a)$. Thus

$$
\begin{aligned}
& \pi\left(c^{-j 2^{i \ell}}\right) P_{(i+1) \ell} f\left(a^{n}\right)-P_{i \ell} f\left(a^{n}\right) \\
& =n\left(\pi\left(c^{-j 2^{i \ell}}\right) P_{(i+1) \ell}-P_{i \ell}\right) Q_{n} f(a) \\
& =n Q_{n}\left(\pi\left(c^{-j 2^{i \ell}}\right) P_{(i+1) \ell}-P_{i \ell}\right) f(a),
\end{aligned}
$$

where the last equality in (19) holds since $c$ is in the center of $\mathbb{H}$, and therefore $Q_{n}$ commutes with all of $\left\{P_{r}\right\}_{r=0}^{\infty}$. Since $\left\|Q_{n}\right\| \leq 1$, it follows from (19) and (18) that

$$
\left\|\pi\left(c^{-j 2^{i \ell}}\right) P_{(i+1) \ell} f\left(a^{n}\right)-P_{i \ell} f\left(a^{n}\right)\right\| \leq \frac{4 K n}{m^{1 / p}} .
$$

Since $f\left(a^{-n}\right)=-\pi(a)^{-n} f\left(a^{n}\right)$, and $\pi(a)$ commutes with $\pi(c)$, and hence with all of $\left\{P_{r}\right\}_{r=0}^{\infty}$, it follows that also

$$
\left\|\pi\left(c^{-j 2^{i \ell}}\right) P_{(i+1) \ell} f\left(a^{-n}\right)-P_{i \ell} f\left(a^{-n}\right)\right\| \leq \frac{4 K n}{m^{1 / p}} .
$$


An identical argument implies the analogous bounds with $a$ replaced by $b$ :

$$
\left\|\pi\left(c^{-j 2^{i \ell}}\right) P_{(i+1) \ell} f\left(b^{n}\right)-P_{i \ell} f\left(b^{n}\right)\right\| \leq \frac{4 K n}{m^{1 / p}}
$$

and

$$
\left\|\pi\left(c^{-j 2^{i \ell}}\right) P_{(i+1) \ell} f\left(b^{-n}\right)-P_{i \ell} f\left(b^{-n}\right)\right\| \leq \frac{4 K n}{m^{1 / p}} .
$$

The cocycle identity implies that for all $n \in \mathbb{N}$,

$$
\begin{aligned}
f\left(c^{n^{2}}\right) & =f\left(\left[a^{n}, b^{n}\right]\right) \\
& =\pi\left(a^{n} b^{n} a^{-n}\right) f\left(b^{-n}\right)+\pi\left(a^{n} b^{n}\right) f\left(a^{-n}\right)+\pi\left(a^{n}\right) f\left(b^{n}\right)+f\left(a^{n}\right) .
\end{aligned}
$$

Thus, using (20), (21), (22) and (23), we conclude the validity of (17).

Lemma 4.2. For every $m, n \in \mathbb{N}$ we have

$$
\left\|P_{m} f\left(c^{n^{2}}\right)\right\| \lesssim \frac{n^{5 / 3}}{2^{m / 3}}
$$

Proof. Note that for every $k \in \mathbb{N}$ we have

$$
P_{m}-\pi\left(c^{k}\right) P_{m}=\frac{1}{2^{m}} \sum_{j=0}^{k-1} \pi(c)^{j}-\frac{1}{2^{m}} \sum_{j=2^{m}}^{2^{m}+k-1} \pi(c)^{j}
$$

Thus,

$$
\left\|P_{m}-\pi\left(c^{k}\right) P_{m}\right\| \leq \frac{2 k}{2^{m}}
$$

The cocycle identity implies that

$$
f\left(c^{(k n)^{2}}\right)=\sum_{j=0}^{k^{2}-1} \pi\left(c^{j n^{2}}\right) f\left(c^{n^{2}}\right) .
$$

Using the fact that $f$ is 1-Lipschitz, $\left\|P_{m}\right\| \leq 1$ and $d_{W}\left(e_{\mathbb{H}}, c^{(k n)^{2}}\right) \leq 4 k n$, we deduce from (26) that

$$
\begin{aligned}
4 k n & \geq\left\|P_{m} f\left(c^{(k n)^{2}}\right)\right\| \\
& \geq \sum_{j=0}^{k^{2}-1}\left(\left\|P_{m} f\left(c^{n^{2}}\right)\right\|-\left\|P_{m}-\pi\left(c^{j n^{2}}\right) P_{m}\right\| \cdot\left\|f\left(c^{n^{2}}\right)\right\|\right) \\
& \stackrel{(25)}{\geq} k^{2}\left\|P_{m} f\left(c^{n^{2}}\right)\right\|-\sum_{j=0}^{k^{2}-1} \frac{2 j n^{2}}{2^{m}} \cdot 4 n \geq k^{2}\left\|P_{m} f\left(c^{n^{2}}\right)\right\|-\frac{4 n^{3} k^{4}}{2^{m}} .
\end{aligned}
$$


Thus,

$$
\left\|P_{m} f\left(c^{n^{2}}\right)\right\| \leq \frac{4 n}{k}+\frac{4 n^{3} k^{2}}{2^{m}} .
$$

Choosing $k=\left\lceil\left(2^{m-1} / n^{2}\right)^{1 / 3}\right\rceil$ in (27) (roughly the optimal choice of $k$ ), we obtain (24).

Lemma 4.3. For every $m, n \in \mathbb{N}$ we have

$$
\left\|f\left(c^{n^{2}}\right)-P_{m} f\left(c^{n^{2}}\right)\right\| \leq 2^{m / 3} n^{1 / 3} .
$$

Proof. In this proof the relation to Section 2 becomes clear. Define $\tilde{f}: \mathbb{H} \rightarrow X$ by

$$
\tilde{f}(h) \stackrel{\text { def }}{=} f(h)-P_{m} f(h)=\left(I-P_{m}\right) f(h) .
$$

Note that $\tilde{f} \in Z^{1}(\pi)$. Fix an integer $k \geq 1$ that will be determined later. Consider the vector $v \in X$ defined by

$$
v \stackrel{\text { def }}{=}-\frac{1}{k} \sum_{j=0}^{k-1} \tilde{f}\left(c^{j}\right)
$$

Then

$$
\|v\| \lesssim \frac{1}{k} \sum_{j=0}^{k-1} \sqrt{j} \lesssim \sqrt{k} .
$$

Since $c$ is in the center of $\mathbb{H}$, we have the following identity for every $h \in \mathbb{H}$ :

$$
\begin{aligned}
-\pi(h) v+\tilde{f}(h) & =\frac{1}{k} \sum_{j=0}^{k-1}\left(\pi(h) \tilde{f}\left(c^{j}\right)+\tilde{f}(h)\right) \\
& =\frac{1}{k} \sum_{j=0}^{k-1} \tilde{f}\left(h c^{j}\right)=\frac{1}{k} \sum_{j=0}^{k-1} \tilde{f}\left(c^{j} h\right) \\
& =\frac{1}{k} \sum_{j=0}^{k-1}\left(\pi\left(c^{j}\right) \tilde{f}(h)+\tilde{f}\left(c^{j}\right)\right)=\frac{1}{k} \sum_{j=0}^{k-1} \pi\left(c^{j}\right) \tilde{f}(h)-v .
\end{aligned}
$$

Note that

$$
\begin{aligned}
\frac{1}{k} \sum_{j=0}^{k-1} \pi\left(c^{j}\right) \tilde{f}(h) & =\frac{1}{k} \sum_{j=0}^{k-1}\left(\pi\left(c^{j}\right) f(h)-\frac{1}{2^{m}} \sum_{i=0}^{2^{m}-1} \pi\left(c^{j+i}\right) f(h)\right) \\
& =\frac{1}{2^{m}} \sum_{i=0}^{2^{m}-1}\left(\frac{1}{k} \sum_{j=0}^{k-1} \pi\left(c^{j}\right)-\frac{1}{k} \sum_{j=i}^{i+k-1} \pi\left(c^{j}\right)\right) f(h) .
\end{aligned}
$$


Hence,

$$
\left\|\frac{1}{k} \sum_{j=0}^{k-1} \pi\left(c^{j}\right) \tilde{f}(h)\right\| \leq \frac{d_{W}\left(h, e_{\mathbb{H}}\right)}{2^{m}} \sum_{i=0}^{2^{m}-1} \frac{2 i}{k} \leq \frac{2^{m}}{k} d_{W}\left(h, e_{\mathbb{H}}\right) .
$$

Combining (30) and (31), we see that $\tilde{f}$ is close to a coboundary in the following sense:

$$
\|\tilde{f}(h)-(\pi(h) v-v)\| \leq \frac{2^{m}}{k} d_{W}\left(h, e_{\mathbb{H}}\right) .
$$

If we now write $c^{n^{2}}=h_{1} h_{2} \cdots h_{4 n}$ for $h_{1}, \ldots, h_{4 n} \in\left\{a, a^{-1}, b, b^{-1}\right\}$, then the cocycle identity for $\tilde{f}$ implies the following bound:

$$
\begin{aligned}
\left\|\tilde{f}\left(c^{n^{2}}\right)\right\| & =\left\|\sum_{i=0}^{4 n-1} \pi\left(h_{1} \cdots h_{4 n-i-1}\right) \tilde{f}\left(h_{4 n-i}\right)\right\| \\
& \stackrel{(32)}{\leq}\left\|\sum_{i=0}^{4 n-1} \pi\left(h_{1} \cdots h_{4 n-i-1}\right)\left(\pi\left(h_{4 n-i}\right) v-v\right)\right\|+\frac{4 n 2^{m}}{k} \\
& =\left\|\pi\left(c^{n^{2}}\right) v-v\right\|+\frac{4 n 2^{m}}{k} \\
& \stackrel{(29)}{ }{ }^{\lesssim} \sqrt{k}+\frac{n 2^{m}}{k} .
\end{aligned}
$$

The optimal choice for $k$ in (33) is $k \asymp n^{2 / 3} 2^{2 m / 3}$. For this choice of $k$, (33) becomes the desired bound (28).

\section{Proof of Theorem 1.2}

As explained in the introduction, using Theorem 9.1 of [21] we may assume without loss of generality that $f \in Z^{1}(\pi)$ for some action $\pi$ of $\mathbb{H}$ on $X$ by linear isometric automorphisms. We may also assume that $t \geq 8^{p}$. Let $m$ be the largest integer such that

$$
m^{m} \leq\left(\frac{t}{4}\right)^{p / 3}
$$

Having defined $m$, let $k$ be the smallest integer such that

$$
m^{\frac{3}{2 p}+\frac{3(k+1)}{p}} \geq t
$$

and set

$$
\ell \stackrel{\text { def }}{=}\left\lceil\frac{6}{p} \log _{2} m\right\rceil
$$


By Lemma 4.1 there exist integers $i \in[k+1, k+m]$ and $j \in\left[0,2^{\ell}-1\right]$ satisfying for all $n \in \mathbb{N}$,

$$
\left\|\pi\left(c^{-j 2^{i \ell}}\right) P_{(i+1) \ell} f\left(c^{n^{2}}\right)-P_{i \ell} f\left(c^{n^{2}}\right)\right\| \leq \frac{16 K n}{m^{1 / p}} .
$$

Choose

$$
n \stackrel{\text { def }}{=} \frac{1}{4}\left\lceil m^{\frac{3}{2 p}} 2^{\frac{i \ell}{2}}\right\rceil
$$

We may write

$$
\begin{aligned}
f\left(c^{n^{2}}\right)= & \pi\left(c^{-j 2^{i \ell}}\right) P_{(i+1) \ell} f\left(c^{n^{2}}\right)+\left(P_{i \ell} f\left(c^{n^{2}}\right)\right. \\
& \left.-\pi\left(c^{-j 2^{i \ell}}\right) P_{(i+1) \ell} f\left(c^{n^{2}}\right)\right)+\left(f\left(c^{n^{2}}\right)-P_{i \ell} f\left(c^{n^{2}}\right)\right) .
\end{aligned}
$$

Hence, by Lemma 4.2, inequality (37), and Lemma 4.3, we obtain the following bound:

$$
\begin{aligned}
\omega_{f}(4 n) & =\omega_{f}\left(d_{W}\left(c^{n^{2}}, e_{\mathbb{H}}\right)\right) \leq\left\|f\left(c^{n^{2}}\right)\right\| \\
& \lesssim \frac{n^{5 / 3}}{2^{(i+1) \ell / 3}}+\frac{8 K n}{m^{1 / p}}+2^{i \ell / 3} n^{1 / 3} \\
& \stackrel{(38) \wedge(36)}{\lesssim} \frac{K n}{m^{1 / p}} .
\end{aligned}
$$

Observe that

$$
4 n \stackrel{(38)}{\geq} m^{\frac{3}{2 p}} 2^{\frac{i \ell}{2}} \geq m^{\frac{3}{2 p}} 2^{\frac{(k+1) \ell}{2}} \stackrel{(36)}{\geq} m^{\frac{3}{2 p}+\frac{3(k+1)}{p}} \stackrel{(35)}{\geq} t .
$$

At the same time,

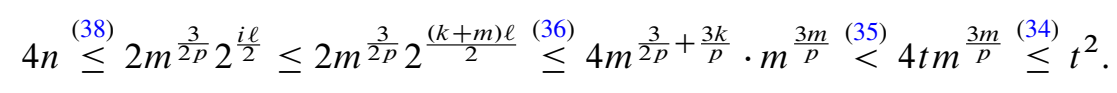

Hence $t \leq 4 n \leq t^{2}$. The definition of $m$ implies that $m \gtrsim \frac{p}{3} \frac{\log n}{\log \log n}$, and therefore (39) becomes:

$$
\frac{\omega_{f}(4 n)}{n} \lesssim K\left(\frac{\log \log n}{\log n}\right)^{1 / p}
$$

The proof of Theorem 1.2 is complete.

\section{Deduction of Theorem 1.3 from Theorem 1.2}

Fix $R \geq 4$ and a function $f: B_{R} \rightarrow X$ satisfying

$$
d_{W}(x, y) \leq\|f(x)-f(y)\| \leq D d_{W}(x, y) \text { for all } x, y \in B_{R} .
$$


Our goal is to bound $D$ from below. Without loss of generality assume that $f(e)=0$. Define $f^{*}: \mathbb{H} \rightarrow X$ by

$$
f^{*}(x)= \begin{cases}f(x), & x \in B_{R / 2}, \\ 2\left(1-\frac{d_{W}(x, e)}{R}\right) f(x), & x \in B_{R} \backslash B_{R / 2}, \\ 0, & x \in \mathbb{H} \backslash B_{R} .\end{cases}
$$

Then $f^{*}$ is $2 D$-Lipschitz and coincides with $f$ on $B_{R / 2}$. Let $\mathcal{N} \subseteq \mathbb{H}$ be a maximal $3 R$-separated subset of $\mathbb{H}$. Thus the function $f^{* *}: \mathbb{H} \rightarrow X$ given by $f^{* *}(x) \stackrel{\text { def }}{=} \sum_{y \in \mathcal{N}} f^{*}\left(y^{-1} x\right)$ (only one summand is nonzero for any given $x$ ) is also $2 D$-Lipschitz.

Fix a free ultrafilter $\mathcal{U}$ on $\mathbb{N}$. Consider the semi-normed space

$$
Y=\left(\ell_{\infty}(\mathbb{H}, X),\|\cdot\|_{Y}\right),
$$

where

$$
\|\psi\|_{Y} \stackrel{\text { def }}{=} \lim _{M \rightarrow u}\left(\frac{1}{\left|B_{M}\right|} \sum_{z \in B_{M}}\|\psi(z)\|^{p}\right)^{1 / p} .
$$

Note that since $X$ satisfies (4), so does $Y . Y$ is a semi-normed space rather than a normed space, so we should formally deal below with the quotient $Y /\{f \in$ $\left.\ell_{\infty}(\mathbb{H}, X):\|f\|_{Y}=0\right\}$, but we will ignore this inessential formality in what follows. (Complete details are as in the proof of Theorem 9.1 in [21]. Alternatively one can note that our proof of Theorem 1.2 carries over without change to the class of semi-normed spaces.)

Define $F: \mathbb{H} \rightarrow Y$ by $F(x)(z) \stackrel{\text { def }}{=} f^{* *}(z x)-f^{* *}(z)$. This is well defined since the metric $d_{W}$ is left-invariant, and therefore $\|F(x)\|_{Y} \leq 2 D d_{W}\left(x, e_{\mathbb{H}}\right)$ for all $x \in \mathbb{H}$. Moreover, by left-invariance, $F$ is $2 D$-Lipschitz. Theorem 1.2 therefore implies that there exist $x, y \in \mathbb{H}$ such that $\sqrt{R / 4} \leq d_{W}(x, y) \leq R / 4$ and

$$
\lim _{M \rightarrow u}\left(\frac{1}{\left|B_{M}\right|} \sum_{z \in B_{M}}\left(\frac{\left\|f^{* *}(z x)-f^{* *}(z y)\right\|}{d_{W}(x, y)}\right)^{p}\right)^{1 / p} \lesssim D K_{p}(X)\left(\frac{\log \log R}{\log R}\right)^{1 / p} .
$$

Fix an integer $M>2 d_{W}(x, e)+4 R$ and write $m=M-2 d_{W}(x, e)-4 R$. Since $\mathcal{N}$ is a maximal $3 R$-separated subset of $\mathbb{H}$, we have $B_{m} x \subseteq \bigcup_{w \in \mathcal{M}} w B_{3 R}$, where $\mathcal{M} \stackrel{\text { def }}{=}\left\{w \in \mathcal{N}: w B_{3 R} \cap B_{m} x \neq \emptyset\right\}$. Hence, since for $r \geq 1$ we have $\left|B_{r}\right| \asymp r^{4}$, we can bound the cardinality of $\mathcal{M}$ as follows:

$$
|\mathcal{M}| \gtrsim\left(\frac{M-2 d_{W}(x, e)-4 R}{3 R}\right)^{4} .
$$

If $w \in \mathcal{M}$ then there exists $z \in B_{m}$ and $g \in B_{3 R}$ such that $z x=w g$. Hence, for 
every $h \in B_{R / 4}$ we have

$$
\begin{aligned}
d_{W}\left(w h x^{-1}, e\right) & =d_{W}\left(z x g^{-1} h x^{-1}, e\right) \\
& \leq d_{W}(z, e)+2 d_{W}(x, e)+d_{W}(g, e)+d_{W}(h, e) \\
& <M
\end{aligned}
$$

Thus the sets $\left\{w B_{R / 4} x^{-1}\right\}_{w \in \mathcal{M}}$ are disjoint and contained in $B_{M}$. Moreover, if $w \in \mathcal{M}$ and $z \in w B_{R / 4} x^{-1}$ then $d_{W}(z x, w) \leq R / 4$, and hence also $d_{W}(z y, w) \leq$ $d_{W}(z y, z x)+d_{W}(z x, w) \leq R / 2$. By the definition of $f^{* *}$, this implies that $f^{* *}(z x)=f\left(w^{-1} z x\right)$ and $f^{* *}(z y)=f\left(w^{-1} z y\right)$.

Hence,

$$
\begin{aligned}
\sum_{z \in B_{M}} & \left(\frac{\left\|f^{* *}(z x)-f^{* *}(z y)\right\|}{d_{W}(x, y)}\right)^{p} \\
& \geq \sum_{w \in \mathcal{M}} \sum_{z \in w B_{R / 4} x^{-1}}\left(\frac{\left\|f\left(w^{-1} z x\right)-\left(w^{-1} z y\right)\right\|}{d_{W}(x, y)}\right)^{p} \\
& \stackrel{(40)}{\geq}|\mathcal{M}| \cdot\left|B_{R / 4}\right| \\
& \stackrel{(43)}{\geq}\left(1-\frac{2 d_{W}(x, e)+4 R}{M}\right)^{4}\left|B_{M}\right| .
\end{aligned}
$$

Theorem 1.3 now follows from (42) and (44).

\section{Embeddings into Hilbert space}

In this section, we prove Theorem 1.4. We will deduce it from an inequality on cocycles for the real Heisenberg group. We switch to the real Heisenberg group because its representation theory is simpler. However, this comes at the cost of adding a (straightforward) discretization step to the proof. The upshot is that we obtain as a byproduct a smooth Poincaré inequality on $\mathbb{H}(\mathbb{R})$ of independent interest; see Theorem 7.5.

The real Heisenberg group $\mathbb{H}(\mathbb{R})$ is defined as the matrix group

$$
\mathbb{H}(\mathbb{R}) \stackrel{\text { def }}{=}\left\{\left(\begin{array}{lll}
1 & u & w \\
0 & 1 & v \\
0 & 0 & 1
\end{array}\right): u, v, w \in \mathbb{R}\right\} .
$$

The discrete Heisenberg group $\mathbb{H}$ sits inside $\mathbb{H}(\mathbb{R})$ as the cocompact discrete subgroup consisting of unipotent matrices with integer coefficients. We equip the group $\mathbb{H}(\mathbb{R})$ with the word metric $d_{S_{\mathbb{R}}}$ associated with the compact symmetric generating set 
$S_{\mathbb{R}}=\left\{a^{u}, b^{v}, c^{w} ;|u|,|v|,|w| \leq 1\right\}$, where

$$
a^{u}=\left(\begin{array}{lll}
1 & u & 0 \\
0 & 1 & 0 \\
0 & 0 & 1
\end{array}\right), \quad b^{v}=\left(\begin{array}{lll}
1 & 0 & 0 \\
0 & 1 & v \\
0 & 0 & 1
\end{array}\right), \quad c^{w}=\left(\begin{array}{ccc}
1 & 0 & w \\
0 & 1 & 0 \\
0 & 0 & 1
\end{array}\right) .
$$

Let $\mu$ denote a Haar measure on $\mathbb{H}(\mathbb{R})$, which coincides with Lebesgue measure under the natural identification of $\mathbb{H}(\mathbb{R})$ with $\mathbb{R}^{3}$.

Theorem 7.1. For every continuous unitary representation $\pi$ of $\mathbb{H}(\mathbb{R})$, any continuous cocycle $\gamma \in Z^{1}(\pi)$ satisfies the inequality

$$
\int_{1}^{\infty} \frac{\left\|\gamma\left(c^{t}\right)\right\|^{2}}{t^{2}} d t \lesssim \int_{-1}^{1}\left(\left\|\gamma\left(a^{u}\right)\right\|^{2}+\left\|\gamma\left(b^{u}\right)\right\|^{2}\right) d u .
$$

Section 7.1 is devoted to the proof of Theorem 7.1. It is clearly enough to check inequality (45) when the representation $\pi$ is irreducible. The proof therefore boils down to a quantitative study of 1-cocycles with values in an irreducible representation of $\mathbb{H}(\mathbb{R})$. In the next three subsections we deduce Theorem 1.4 from Theorem 7.1 by a succession of reductions. Finally, in the last subsection, we state a smooth Poincaré inequality on the real Heisenberg group, whose proof, being very similar to the discrete one, is explained in a few sentences.

7.1. Proof of Theorem 7.1. By the Stone-von Neumann theorem (see for example Chapter 2 in [12]), irreducible representations of $\mathbb{H}(\mathbb{R})$ are of two types: those that factor through the center, and, for every $\lambda \in \mathbb{R} \backslash\{0\}$, the representation $\pi_{\lambda}$ on $L_{2}(\mathbb{R})$ satisfying

$$
\pi_{\lambda}\left(a^{u} b^{v} c^{w}\right)(h)(x) \stackrel{\text { def }}{=} e^{2 \pi i \lambda v x} h(x+u) e^{2 \pi i \lambda w} \quad \text { for all } h \in L_{2}(\mathbb{R}) .
$$

Note that if a nontrivial irreducible representation $\pi$ factors through the center then any 1-cocycle $\gamma \in Z^{1}(\pi)$ must vanish on the center. Indeed, $\gamma\left(c^{w}\right)$ is invariant under $\pi(\mathbb{H})$ for every $w \in \mathbb{R}$, which, since the representation is supposed to be irreducible and nontrivial, implies that $\gamma\left(c^{w}\right)=0$. Therefore, in proving Theorem 7.1 we may assume that $\pi=\pi_{\lambda}$ for some $\lambda \neq 0$.

By Theorem 7 of [14] all 1-cocycles $\gamma \in Z^{1}\left(\pi_{\lambda}\right)$ can be approximated uniformly on compact sets by coboundaries. Hence, it is enough to consider the case where $\gamma$ is of the form $\gamma(x)=\pi_{\lambda}(x) h-h$, for some $h \in L_{2}(\mathbb{R})$. We may assume that $\|h\|=1$. By the definition (46), for every $w \in \mathbb{R}$ we have $\left\|\gamma\left(c^{w}\right)\right\|^{2}=4 \sin ^{2}(\pi \lambda w)$, from which we deduce that

$$
\int_{1}^{\infty} \frac{\left\|\gamma\left(c^{w}\right)\right\|^{2}}{w^{2}} d w \lesssim \int_{1}^{\infty} \frac{\sin ^{2}(\pi \lambda w)}{w^{2}} d w=|\lambda| \int_{|\lambda|}^{\infty} \frac{\sin ^{2}(\pi w)}{w^{2}} d w \lesssim \min \{|\lambda|, 1\} .
$$


Also,

$$
\begin{aligned}
\int_{-1}^{1}( & \left.\left\|\gamma\left(a^{u}\right)\right\|^{2}+\left\|\gamma\left(b^{u}\right)\right\|^{2}\right) d u \\
& =\int_{-1}^{1} \int_{\mathbb{R}}\left(|h(x+u)-h(x)|^{2}+2|h(x)|^{2}(1-\cos (2 \pi \lambda u x))\right) d x d u \\
& \asymp \int_{-1}^{1} \int_{\mathbb{R}}|h(x+u)-h(x)|^{2} d x d u+\int_{\mathbb{R}}|h(x)|^{2} \min \left\{\lambda^{2} x^{2}, 1\right\} d x .
\end{aligned}
$$

Since $\mathbb{R}=(\mathbb{R} \backslash[-|u|,|u|]) \cup(u+(\mathbb{R} \backslash[-|u|,|u|])) \cup(-u+(\mathbb{R} \backslash[-|u|,|u|]))$ for every $u \in \mathbb{R}$, we can bound $\|h\|^{2}=1$ from above as follows.

$$
\begin{aligned}
1 & \leq \int_{\mathbb{R} \backslash[-|u|,|u|]}\left(|h(x+u)|^{2}+|h(x-u)|^{2}+|h(x)|^{2}\right) d x \\
& \lesssim \int_{\mathbb{R}}\left(|h(x+u)-h(x)|^{2}+|h(x)-h(x-u)|^{2}\right) d x+\int_{\mathbb{R} \backslash[-|u|,|u|]}|h(x)|^{2} d x \\
& \leq \int_{\mathbb{R}}\left(|h(x+u)-h(x)|^{2}+|h(x)-h(x-u)|^{2}\right) d x \\
& \quad+\frac{1}{\min \left\{\lambda^{2} u^{2}, 1\right\}} \int_{\mathbb{R}}|h(x)|^{2} \min \left\{\lambda^{2} x^{2}, 1\right\} d x .
\end{aligned}
$$

Write $k=\lceil 1 / \sqrt{|\lambda|}\rceil$. By applying (49) with $u=k v$, and integrating over $v \in$ $[-1,-1 / 2] \cup[1 / 2,1]$, we see that

$$
\begin{aligned}
& \int_{1}^{\infty} \frac{\left\|\gamma\left(c^{w}\right)\right\|^{2}}{w^{2}} d w \\
& \text { (47) } \\
& \stackrel{\min }{\lesssim}\{|\lambda|, 1\} \\
& \stackrel{(49)}{\lesssim} \min \{|\lambda|, 1\} \int_{-1}^{1} \int_{\mathbb{R}}|h(x+k v)-h(x)|^{2} d x d v \\
& +\frac{\min \{|\lambda|, 1\}}{\min \left\{\lambda^{2}\lceil 1 / \sqrt{|\lambda|}\rceil^{2}, 1\right\}} \int_{\mathbb{R}}|h(x)|^{2} \min \left\{\lambda^{2} x^{2}, 1\right\} d x \\
& \lesssim \min \{|\lambda|, 1\} k \sum_{j=1}^{k} \int_{-1}^{1} \int_{\mathbb{R}}|h(x+j v)-h(x+(j-1) v)|^{2} d x d v \\
& +\int_{\mathbb{R}}|h(x)|^{2} \min \left\{\lambda^{2} x^{2}, 1\right\} d x \\
& =\min \{|\lambda|, 1\} k^{2} \int_{-1}^{1} \int_{\mathbb{R}}|h(x+u)-h(x)|^{2} d x d u+\int_{\mathbb{R}}|h(x)|^{2} \min \left\{\lambda^{2} x^{2}, 1\right\} d x \\
& \stackrel{(48)}{\lesssim} \int_{-1}^{1}\left(\left\|\gamma\left(a^{u}\right)\right\|^{2}+\left\|\gamma\left(b^{u}\right)\right\|^{2}\right) d u \text {. }
\end{aligned}
$$

The proof of Theorem 7.1 is complete. 


\subsection{Reduction to finitely supported functions}

Claim 7.2. Inequality (8) is a consequence of the following statement. For every finitely supported $\phi: \mathbb{H} \rightarrow L_{2}$, we have

$$
\sum_{x \in \mathbb{H}} \sum_{k=1}^{\infty} \frac{\left\|\phi\left(x c^{k}\right)-\phi(x)\right\|_{2}^{2}}{k^{2}} \lesssim \sum_{x \in \mathbb{H}}\left(\|\phi(x a)-\phi(x)\|_{2}^{2}+\|\phi(x b)-\phi(x)\|_{2}^{2}\right) .
$$

Proof. Fix $R \in \mathbb{N}$ and $f: \mathbb{H} \rightarrow L_{2}$. Note that since (8) is not sensitive to adding a constant to the function $f$, we can assume without loss of generality that the average of $f$ over $B_{7 R}$ is zero.

Define a cutoff function $\xi: \mathbb{H} \rightarrow[0,1]$ by

$$
\xi(x) \stackrel{\text { def }}{=} \begin{cases}1, & x \in B_{5 R}, \\ 6-\frac{d_{W}(x, e)}{R}, & x \in B_{6 R} \backslash B_{5 R}, \\ 0, & x \in \mathbb{H} \backslash B_{6 R}\end{cases}
$$

and let $\phi \stackrel{\text { def }}{=} \xi f$. Then $\phi$ is supported on $B_{6 R}$. Since $\xi$ is $1 / R$-Lipschitz and takes values in $[0,1]$, for all $x \in \mathbb{H}$ and $s \in S$,

$$
\begin{aligned}
\|\phi(x)-\phi(x s)\|_{2}^{2} & \lesssim|\xi(x)-\xi(x s)|^{2} \cdot\|f(x)\|_{2}^{2}+|\xi(x s)|^{2} \cdot\|f(x)-f(x s)\|_{2}^{2} \\
& \leq \frac{1}{R^{2}}\|f(x)\|^{2}+\|f(x)-f(x s)\|_{2}^{2} .
\end{aligned}
$$

Note that if $k \in\left\{1, \ldots, R^{2}\right\}$ then $d_{W}\left(e, c^{k}\right) \leq 4 R$, and hence for $x \in B_{R}$ we have $x c^{k} \in B_{5 R}$. Therefore, an application of (50) to $\phi$ yields the estimate

$$
\begin{aligned}
\sum_{x \in B_{R}} & \sum_{k=1}^{R^{2}} \frac{\left\|f\left(x c^{k}\right)-f(x)\right\|_{2}^{2}}{k^{2}} \\
& \leq \sum_{x \in \mathbb{H}} \sum_{k=1}^{\infty} \frac{\left\|\phi\left(x c^{k}\right)-\phi(x)\right\|_{2}^{2}}{k^{2}} \\
& \lesssim \sum_{x \in \mathbb{H}} \max _{s \in S}\|\phi(x s)-\phi(x)\|_{2}^{2} \\
& =\sum_{x \in B_{7 R}} \max _{s \in S}\|\phi(x s)-\phi(x)\|_{2}^{2} \\
& \stackrel{(51)}{\leq} \frac{1}{R^{2}} \sum_{x \in B_{7 R}}\|f(x)\|_{2}^{2}+\sum_{x \in B_{7 R}} \max _{s \in S}\|f(x s)-f(x)\|_{2}^{2} .
\end{aligned}
$$


By [17], Theorem 2.2 (a discrete version of the classical Heisenberg local Poincaré inequality [16]),

$$
\frac{1}{R^{2}} \sum_{x \in B_{7 R}}\|f(x)\|_{2}^{2} \lesssim \sum_{x \in B_{22 R}}\left(\|f(x a)-f(x)\|_{2}^{2}+\|f(x b)-f(x)\|_{2}^{2}\right),
$$

where we used the fact that the average of $f$ on $B_{7 R}$ vanishes. The desired inequality (8) is now a consequence of (52) and (53).

\subsection{Reduction to an inequality on the real Heisenberg group}

Claim 7.3. Inequality (50) is a consequence of the following statement. For every continuous and compactly supported function $\psi: \mathbb{H}(\mathbb{R}) \rightarrow L_{2}$, we have

$$
\int_{\mathbb{H}(\mathbb{R})} \int_{1}^{\infty} \frac{\left\|\psi\left(x c^{t}\right)-\psi(x)\right\|_{2}^{2}}{t^{2}} d t d \mu(x) \lesssim \int_{\mathbb{H}(\mathbb{R})}\left(\sup _{s \in S_{\mathbb{R}}}\|\psi(x s)-\psi(x)\|_{2}^{2}\right) d \mu(x) .
$$

Proof. For $r>0$ let $B_{r}^{\mathbb{R}} \subseteq \mathbb{H}(\mathbb{R})$ denote the ball of radius $r$ with respect to the metric $d_{S_{\mathbb{R}}}$. Note that $\mathbb{H}(\mathbb{R})=\bigcup_{g \in \mathbb{H}} g B_{2}^{\mathbb{R}}$. Let $\sigma: \mathbb{H}(\mathbb{R}) \rightarrow[0,1]$ be a continuous nonnegative function, which equals 1 on $B_{2}^{\mathbb{R}}$ and 0 outside of $B_{3}^{\mathbb{R}}$. Let $\tilde{\sigma}=\sum_{g \in \mathbb{H}} \sigma_{g}$, where $\sigma_{g}(x)=\sigma\left(g^{-1} x\right)$. For all $x \in \mathbb{H}(\mathbb{R})$ we have $1 \leq \tilde{\sigma}(x) \leq C$ for some $C \in(0, \infty)$. Writing $\beta=\sigma / \tilde{\sigma}$ and $\beta_{g}(x)=\beta\left(g^{-1} x\right)$, we see that $\left\{\beta_{g}\right\}_{g \in \mathbb{H}}$ is a continuous partition of unity for $\mathbb{H}(\mathbb{R})$ satisfying

$$
\sup _{x \in \mathbb{H}(\mathbb{R})} \sum_{g \in \mathbb{H}} \sup _{s \in S_{\mathbb{R}}}\left|\beta_{g}(x s)-\beta_{g}(x)\right|<\infty .
$$

Throughout the ensuing argument we will use repeatedly the fact that for every $x \in \mathbb{H}(\mathbb{R})$ the number of elements $g \in \mathbb{H}$ for which $\beta_{g}(x)>0$ is bounded by a

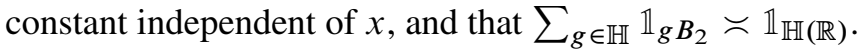

Let $\phi: \mathbb{H} \rightarrow L_{2}$ be a finitely supported function on the discrete Heisenberg group $\mathbb{H}$. Define a function on the real Heisenberg group $\mathbb{H}(\mathbb{R})$ by

$$
\psi(x)=\sum_{g \in \mathbb{H}} \phi(g) \beta_{g}(x) .
$$

Then $\psi$ is compactly supported. We will eventually apply (54) to $\psi$, but before doing so we will need some preparatory estimates.

The metric $d_{S_{\mathbb{R}}}$ restricted to $\mathbb{H} \subseteq \mathbb{H}(\mathbb{R})$ is bi-Lipschitz equivalent to $d_{W}$ [5], Theorem 8.3.19. It follows that for all $g_{0} \in \mathbb{H}$, if $x \in g_{0} B_{3}^{\mathbb{R}}$ then the sum $\psi(x)-$ $\phi\left(g_{0}\right)=\sum_{g \in \mathbb{H}} \beta_{g}(x)\left(\phi(g)-\phi\left(g_{0}\right)\right)$ is supported on elements of the form $g=g_{0} h$, with $d_{W}(h, e) \leq K$, for some universal constant $K \in \mathbb{N}$. Thus, using (55) we see that for all $x \in g_{0} B_{2}^{\mathbb{R}}$,

$$
\sup _{s \in S_{\mathbb{R}}}\|\psi(x s)-\psi(x)\|_{2}^{2} \lesssim \sum_{h \in B_{K}}\left\|\phi\left(g_{0} h\right)-\phi\left(g_{0}\right)\right\|_{2}^{2} .
$$


Integrating (56) over $g_{0} B_{2}^{\mathbb{R}}$ gives the following inequality:

$$
\int_{g_{0} B_{2}^{\mathbb{R}}}\left(\sup _{s \in S_{\mathbb{R}}}\|\psi(x s)-\psi(x)\|_{2}^{2}\right) d \mu(x) \lesssim \sum_{h \in B_{K}}\left\|\phi\left(g_{0} h\right)-\phi\left(g_{0}\right)\right\|_{2}^{2} .
$$

By summing (57) over $g_{0} \in \mathbb{H}$ we see that

$$
\int_{\mathbb{H}(\mathbb{R})}\left(\sup _{s \in S_{\mathbb{R}}}\|\psi(x s)-\psi(x)\|_{2}^{2}\right) d \mu(x) \lesssim \sum_{g \in \mathbb{H}} \max _{s \in S}\|\phi(g)-\phi(g s)\|_{2}^{2},
$$

where we used the bound

$$
\sum_{g_{0} \in \mathbb{H}} \sum_{h \in B_{K}}\left\|\phi\left(g_{0} h\right)-\phi\left(g_{0}\right)\right\|_{2}^{2} \lesssim \sum_{g \in \mathbb{H}} \max _{s \in S}\|\phi(g)-\phi(g s)\|_{2}^{2},
$$

which follows by writing each $h \in B_{K}$ as a product of at most $K$ elements of $S$, and using the triangle inequality.

In order to deduce from (54) a corresponding bound on $\phi$, we need to bound $\phi$ in terms of $\psi$. To this end, note that for all $g_{0} \in \mathbb{H}$,

$$
\begin{aligned}
\psi\left(g_{0}\right) & =\phi\left(g_{0}\right)+\sum_{g \in \mathbb{H}} \beta\left(g^{-1} g_{0}\right)\left(\phi(g)-\phi\left(g_{0}\right)\right) \\
& =\phi\left(g_{0}\right)+\sum_{h \in B_{K}} \beta\left(h^{-1}\right)\left(\phi\left(g_{0} h\right)-\phi\left(g_{0}\right)\right) .
\end{aligned}
$$

It follows that for all $g_{0}, g_{1} \in \mathbb{H}$ we have

$$
\begin{aligned}
& \left\|\phi\left(g_{0}\right)-\phi\left(g_{1}\right)\right\|_{2}^{2} \\
& \quad \lesssim\left\|\psi\left(g_{0}\right)-\psi\left(g_{1}\right)\right\|_{2}^{2}+\sum_{h \in B_{K}}\left(\left\|\phi\left(g_{0}\right)-\phi\left(g_{0} h\right)\right\|_{2}^{2}+\left\|\phi\left(g_{1}\right)-\phi\left(g_{1} h\right)\right\|_{2}^{2}\right) .
\end{aligned}
$$

If $x, y \in \mathbb{H}(\mathbb{R})$ satisfy $\max \left\{d_{S_{\mathbb{R}}}\left(x, g_{0}\right), d_{S_{\mathbb{R}}}\left(y, g_{1}\right)\right\} \leq 2$, then using (56) we deduce from (60) that

$$
\begin{aligned}
& \left\|\phi\left(g_{0}\right)-\phi\left(g_{1}\right)\right\|_{2}^{2} \\
& \quad \lesssim\|\psi(x)-\psi(y)\|_{2}^{2}+\sum_{h \in B_{K}}\left(\left\|\phi\left(g_{0}\right)-\phi\left(g_{0} h\right)\right\|_{2}^{2}+\left\|\phi\left(g_{1}\right)-\phi\left(g_{1} h\right)\right\|_{2}^{2}\right) .
\end{aligned}
$$

Fix $g_{0} \in \mathbb{H}, x \in g_{0} B_{1}^{\mathbb{R}}, k \in \mathbb{N}$ and $t \in[k, k+1]$. Writing $g_{1}=g_{0} c^{k}$ and $y=x c^{t}$, we have $d_{S_{\mathbb{R}}}\left(x, g_{0}\right) \leq 1$ and $d_{S_{\mathbb{R}}}\left(y, g_{1}\right)=d_{S_{\mathbb{R}}}\left(c^{-k} g_{0}^{-1} x c^{t}, e\right)=$ 
$d_{S_{\mathbb{R}}}\left(g_{0}^{-1} x c^{t-k}, e\right) \leq d_{S_{\mathbb{R}}}\left(g_{0}^{-1} x, e\right)+d_{S_{\mathbb{R}}}\left(c^{t-k}, e\right) \leq 2$. We may therefore apply (61) and deduce that for all $g_{0} \in \mathbb{H}, x \in g_{0} B_{1}^{\mathbb{R}}$ and $k \in \mathbb{N}$,

$$
\begin{aligned}
& \left\|\phi\left(g_{0} c^{k}\right)-\phi\left(g_{0}\right)\right\|_{2}^{2} \\
& \lesssim\left\|\psi\left(x c^{t}\right)-\psi(x)\right\|_{2}^{2} \\
& \quad+\sum_{h \in B_{K}}\left(\left\|\phi\left(g_{0}\right)-\phi\left(g_{0} h\right)\right\|_{2}^{2}+\left\|\phi\left(g_{0} c^{k}\right)-\phi\left(g_{0} c^{k} h\right)\right\|_{2}^{2}\right) .
\end{aligned}
$$

Integrating (62) over $x \in g_{0} B_{1}^{\mathbb{R}}$ and $t \in[k, k+1]$, we see that

$$
\begin{aligned}
& \frac{\left\|\phi\left(g_{0} c^{k}\right)-\phi\left(g_{0}\right)\right\|_{2}^{2}}{k^{2}} \\
& \quad \lesssim \int_{g_{0} B_{1}^{\mathbb{R}}} \int_{k}^{k+1} \frac{\left\|\psi\left(x c^{t}\right)-\psi(x)\right\|_{2}^{2}}{t^{2}} d t d \mu(x) \\
& \quad+\frac{1}{k^{2}} \sum_{h \in B_{K}}\left(\left\|\phi\left(g_{0}\right)-\phi\left(g_{0} h\right)\right\|_{2}^{2}+\left\|\phi\left(g_{0} c^{k}\right)-\phi\left(g_{0} c^{k} h\right)\right\|_{2}^{2}\right) .
\end{aligned}
$$

Since $g_{0} B_{1}^{\mathbb{R}}$ and $g_{0}^{\prime} B_{1}^{\mathbb{R}}$ intersect at a set of measure zero if $g_{0} \neq g_{0}^{\prime}$, by summing (63) over $g_{0} \in \mathbb{H}$ and $k \in \mathbb{N}$, we see that

$$
\begin{aligned}
& \sum_{g_{0} \in \mathbb{H}} \sum_{k=1}^{\infty} \frac{\left\|\phi\left(g_{0} c^{k}\right)-\phi\left(g_{0}\right)\right\|_{2}^{2}}{k^{2}} \\
& \quad \lesssim \int_{\mathbb{H}(\mathbb{R})} \int_{1}^{\infty} \frac{\left\|\psi\left(x c^{t}\right)-\psi(x)\right\|_{2}^{2}}{t^{2}} d t d \mu(x)+\sum_{g_{0} \in \mathbb{H}} \sum_{h \in B_{K}}\left\|\phi\left(g_{0}\right)-\phi\left(g_{0} h\right)\right\|_{2}^{2} \\
& \quad \stackrel{(59)}{\lesssim} \int_{\mathbb{H}(\mathbb{R})} \int_{1}^{\infty} \frac{\left\|\psi\left(x c^{t}\right)-\psi(x)\right\|_{2}^{2}}{t^{2}} d t d \mu(x)+\sum_{g \in \mathbb{H}} \max _{s \in S}\|\phi(g)-\phi(g s)\|_{2}^{2} \\
& \stackrel{(54)}{\lesssim} \int_{\mathbb{H}(\mathbb{R})}\left(\sup _{s \in S_{\mathbb{R}}}\|\psi(x s)-\psi(x)\|_{2}^{2}\right) d \mu(x)+\sum_{g \in \mathbb{H}} \max _{s \in S}\|\phi(g)-\phi(g s)\|_{2}^{2} \\
& \stackrel{(58)}{\lesssim} \sum_{g \in \mathbb{H}} \max _{s \in S}\|\phi(g)-\phi(g s)\|_{2}^{2} .
\end{aligned}
$$

\subsection{Reduction to a 1-cocycle on $\mathbb{H}(\mathbb{R})$}

Claim 7.4. Inequality (54) follows from Theorem 7.1.

Proof. Let $\psi: \mathbb{H}(\mathbb{R}) \rightarrow L_{2}$ be continuous and supported in $B_{r}^{\mathbb{R}}$ for some $r \geq 1$. Take a maximal family $\left\{x_{i} B_{10 r}^{\mathbb{R}}\right\}_{i=1}^{\infty}$ of disjoint balls of radius $10 r$. Define $\varphi: \mathbb{H}(\mathbb{R}) \rightarrow L_{2}$ 
by $\varphi(g)=\sum_{i=1}^{\infty} \psi\left(x_{i}^{-1} g\right)$. Note that since $d_{S_{\mathbb{R}}}\left(c^{r^{2}}, e\right) \leq 4 r$, for each $i \in \mathbb{N}$ we have

$$
\begin{aligned}
\int_{\mathbb{H}(\mathbb{R})} & \int_{1}^{\infty} \frac{\left\|\psi\left(x c^{t}\right)-\psi(x)\right\|_{2}^{2}}{t^{2}} d t d \mu(x) \\
= & \int_{x_{i} B_{5 r}^{\mathbb{R}}} \int_{1}^{r^{2}} \frac{\left\|\varphi\left(x c^{t}\right)-\varphi(x)\right\|_{2}^{2}}{t^{2}} d t d \mu(x) .
\end{aligned}
$$

Similarly,

$$
\int_{\mathbb{H}(\mathbb{R})}\left(\sup _{s \in S_{\mathbb{R}}}\|\psi(x s)-\psi(x)\|_{2}^{2}\right) d \mu(x)=\int_{x_{i} B_{2 r}^{\mathbb{R}}}\left(\sup _{s \in S_{\mathbb{R}}}\|\varphi(x s)-\varphi(x)\|_{2}^{2}\right) d \mu(x) .
$$

Let $X$ be the space of all finitely supported complex-valued functions on $\mathbb{H}(\mathbb{R})$. We denote by $\pi$ the action of $\mathbb{H}(\mathbb{R})$ on $X$ given by $\pi(x) \delta_{y} \stackrel{\text { def }}{=} \delta_{x y}$, where $\delta_{z}: \mathbb{H}(\mathbb{R}) \rightarrow$ $\mathbb{C}$ denotes the function which equals 1 at $z \in \mathbb{H}(\mathbb{R})$ and equals 0 elsewhere. Let $\mathcal{U}$ be a free ultrafilter on $\mathbb{N}$. Define a scalar product $[\cdot, \cdot]$ on $X$ by:

$$
\left[\delta_{x}, \delta_{y}\right] \stackrel{\text { def }}{=} \lim _{n \rightarrow u} \frac{1}{\mu\left(B_{n}^{\mathbb{R}}\right)} \int_{B_{n}^{\mathbb{R}}}\langle\varphi(z x), \varphi(z y)\rangle d \mu(z),
$$

where $\langle\cdot, \cdot\rangle$ denotes the scalar product on $L_{2}$.

Since $\left\{B_{n}\right\}_{n=1}^{\infty}$ is a Følner sequence for $\mathbb{H}(\mathbb{R})$, a limit along a free ultrafilter of averages over $B_{n}$ when $n \rightarrow \infty$ is an invariant mean on $\mathbb{H}(\mathbb{R})$. It follows that the scalar product defined in $(66)$ is $\pi(\mathbb{H}(\mathbb{R}))$-invariant. Thus $\pi$ is a unitary representation of $\mathbb{H}(\mathbb{R})$ (formally we should first pass to the completion of the quotient of $X$ by the subspace consisting of norm-zero elements, but we will ignore this inessential point in what follows). We note that $\pi$ is also continuous in the strong operator topology. Indeed, since $\psi$ is continuous and compactly supported, $\varphi$ is uniformly continuous. Thus, writing $\|f\|_{X}^{2} \stackrel{\text { def }}{=}[f, f]$ for $f \in X$, we have for every $y \in \mathbb{H}(\mathbb{R})$,

$$
\begin{aligned}
\lim _{x \rightarrow e}\left\|\pi(x) \delta_{y}-\delta_{y}\right\|_{X}^{2} & =\lim _{x \rightarrow e} \lim _{n \rightarrow u} \frac{1}{\mu\left(B_{n}^{\mathbb{R}}\right)} \int_{B_{n}^{\mathbb{R}}}\|\varphi(g x y)-\varphi(g y)\|_{2}^{2} d \mu(g) \\
& \leq \lim _{x \rightarrow e} \sup _{g \in \mathbb{H}(\mathbb{R})}\|\varphi(g x y)-\varphi(g y)\|_{2}^{2}=0,
\end{aligned}
$$

implying the strong continuity of $\pi$.

Let $\gamma: \mathbb{H}(\mathbb{R}) \rightarrow X$ be given by $\gamma(x)=\delta_{x}-\delta_{e}$. Then $\pi \in Z^{1}(\pi)$ and for all $x \in X$,

$$
\|\gamma(x)\|_{X}^{2}=\lim _{n \rightarrow u} \frac{1}{\mu\left(B_{n}^{\mathbb{R}}\right)} \int_{B_{n}^{\mathbb{R}}}\|\varphi(g x)-\varphi(g)\|_{2}^{2} d \mu(g) .
$$

Arguing as in (67), the uniform continuity of $\varphi$ and (68) imply that $\gamma$ is a continuous 1 -cocycle. 
Fix $n>100 r$ large enough so as to ensure that we have $\mu\left(B_{n} \backslash B_{n-100 r}\right) \leq$ $\mu\left(B_{n}\right) / 2$. Define $I=\left\{i \in \mathbb{N}: x_{i} B_{5 r}^{\mathbb{R}} \subseteq B_{n}\right\}$ and write $\Omega=\bigcup_{i \in I} x_{i} B_{5 r}^{\mathbb{R}}$ and $\Omega^{\prime}=\bigcup_{i \in I} x_{i} B_{10 r}^{\mathbb{R}}$. By the maximality of $\left\{x_{i} B_{10 r}^{\mathbb{R}}\right\}_{i=1}^{\infty}$ we have $\Omega^{\prime} \supseteq B_{n-100 r}$. Hence $\mu\left(\Omega^{\prime}\right) \geq \mu\left(B_{n}\right) / 2$. Since $\mathbb{H}(\mathbb{R})$ is doubling, $\mu\left(\Omega^{\prime}\right) \lesssim \mu(\Omega)$, and therefore $|I| \mu\left(B_{5 r}^{\mathbb{R}}\right) \geq \mu(\Omega) \gtrsim \mu\left(B_{n}\right)$. Note that if $g \in x_{i} B_{5 r}^{\mathbb{R}}$ for some $i \in \mathbb{N}$ then for every $t \in\left[1, r^{2}\right]$ we have $g c^{t} \in x_{i} B_{10 r}$. Hence,

$$
\begin{aligned}
& \frac{1}{\mu\left(B_{n}^{\mathbb{R}}\right)} \int_{B_{n}^{\mathbb{R}}}\left\|\varphi\left(g c^{t}\right)-\varphi(g)\right\|_{2}^{2} d \mu(g) \\
& \quad \geq \frac{1}{\mu\left(B_{n}^{\mathbb{R}}\right)} \sum_{i \in I} \int_{x_{i} B_{5 r}^{\mathbb{R}}}\left\|\varphi\left(g c^{t}\right)-\varphi(g)\right\|_{2}^{2} d \mu(g) \\
& \frac{|I|}{\mu\left(B_{n}^{\mathbb{R}}\right)} \int_{B_{5 r}^{\mathbb{R}}}\left\|\psi\left(x c^{t}\right)-\psi(x)\right\|_{2}^{2} d \mu(x) \\
& \gtrsim \frac{1}{\mu\left(B_{5 r}^{\mathbb{R}}\right)} \int_{B_{5 r}^{\mathbb{R}}}\left\|\psi\left(x c^{t}\right)-\psi(x)\right\|_{2}^{2} d \mu(x) .
\end{aligned}
$$

It follows from (68) and (69) that

$$
\begin{aligned}
& \frac{1}{\mu\left(B_{5 r}^{\mathbb{R}}\right)} \int_{B_{5 r}^{\mathbb{R}}} \int_{1}^{r^{2}} \frac{\left\|\psi\left(x c^{t}\right)-\psi(x)\right\|_{2}^{2}}{t^{2}} d \mu(x) \\
& \quad \lesssim \int_{1}^{r^{2}} \frac{\left\|\gamma\left(c^{t}\right)\right\|_{X}^{2}}{t^{2}} d t \\
& \quad \stackrel{(45)}{\lesssim} \sup _{s \in S_{\mathbb{R}}}\|\gamma(s)\|_{X}^{2} \\
& \quad \stackrel{(68)}{=} \sup _{s \in S_{\mathbb{R}}} \lim _{n \rightarrow u} \frac{1}{\mu\left(B_{n}^{\mathbb{R}}\right)} \int_{B_{n}^{\mathbb{R}}}\|\varphi(g s)-\varphi(g)\|_{2}^{2} d \mu(g) .
\end{aligned}
$$

Let $J \subseteq \mathbb{N}$ denote the set of $i \in \mathbb{N}$ such that $B_{n} \cap x_{i} B_{2 r} \neq \emptyset$. Then $|J| \mu\left(B_{2 r}^{\mathbb{R}}\right) \leq$ $\mu\left(B_{n+4 r}^{\mathbb{R}}\right)$. It follows that for all $s \in S_{\mathbb{R}}$,

$$
\begin{aligned}
\int_{B_{n}^{\mathbb{R}}}\|\varphi(g s)-\varphi(g)\|_{2}^{2} d \mu(g) & \leq \sum_{i \in J} \int_{x_{i} B_{2 r}}\|\varphi(g s)-\varphi(g)\|_{2}^{2} d \mu(g) \\
& =|J| \int_{B_{2 r}}\|\psi(x s)-\psi(x)\|_{2}^{2} d \mu(x) \\
& \leq \frac{\mu\left(B_{n+4 r}^{\mathbb{R}}\right)}{\mu\left(B_{2 r}^{\mathbb{R}}\right)} \int_{B_{2 r}}\|\psi(x s)-\psi(x)\|_{2}^{2} d \mu(x) .
\end{aligned}
$$


Substituting (71) into (70) we conclude that

$$
\begin{aligned}
& \int_{\mathbb{H}(\mathbb{R})} \int_{1}^{\infty} \frac{\left\|\psi\left(x c^{t}\right)-\psi(x)\right\|_{2}^{2}}{t^{2}} d t d \mu(x) \\
& \stackrel{(64)}{=} \int_{B_{5 r}^{\mathbb{R}}} \int_{1}^{r^{2}} \frac{\left\|\psi\left(x c^{t}\right)-\psi(x)\right\|_{2}^{2}}{t^{2}} d \mu(x) \\
& \stackrel{(70) \wedge(71)}{\lesssim} \frac{\mu\left(B_{5 r}^{\mathbb{R}}\right)}{\mu\left(B_{2 r}^{\mathbb{R}}\right)} \sup _{s \in S_{\mathbb{R}}} \lim _{n \rightarrow u} \frac{\mu\left(B_{n+4 r}^{\mathbb{R}}\right)}{\mu\left(B_{n}^{\mathbb{R}}\right)} \int_{B_{2 r}}\|\psi(x s)-\psi(x)\|_{2}^{2} d \mu(x) \\
& \stackrel{(65)}{\lesssim} \int_{\mathbb{H}(\mathbb{R})}\left(\sup _{s \in S_{\mathbb{R}}}\|\psi(x s)-\psi(x)\|_{2}^{2}\right) d \mu(x)
\end{aligned}
$$

This completes the proof of Claim 7.4, and therefore also the proof of Theorem 1.4.

7.5. A smooth Poincaré inequality on $\mathbb{H}(\mathbb{R})$. Equip $\mathbb{H}(\mathbb{R})$ with the left-invariant Riemannian metric given by $d u^{2}+d v^{2}+(d w-u d v)^{2}$. In what follows, given a smooth function $f: \mathbb{H}(\mathbb{R}) \rightarrow \mathbb{R}$ we let $\nabla_{\mathbb{H}} f$ denote its gradient with respect to this Riemannian structure.

Theorem 7.5. For every smooth function $f: \mathbb{H}(\mathbb{R}) \rightarrow \mathbb{R}$, and all $R>0$,

$$
\int_{B} \int_{R}^{R^{2}} \frac{\left|f\left(x c^{t}\right)-f(x)\right|^{2}}{t^{2}} d t d \mu(x) \lesssim \int_{B_{C R}^{\mathbb{R}}}\left|\nabla_{\mathbb{H}} f(x)\right|^{2} d \mu(x),
$$

where $C>0$ is a universal constant.

The proof of this Poincaré inequality can be obtained from Theorem 7.1 in a way similar, and actually even shorter than its discrete counterpart. Indeed, the discretization step of Claim 7.3 is not needed here. The other difference lies in the first step, where instead of the discrete Poincaré inequality (53), we use the following smooth version, which is due to [16]. For all $R>0$, and for all smooth functions $f: \mathbb{H}(\mathbb{R}) \rightarrow \mathbb{R}$ whose integral over $B_{R}^{\mathbb{R}}$ is zero,

$$
\frac{1}{R^{2}} \int_{B_{R}^{\mathbb{R}}}|f(g)|^{2} d \mu(g) \lesssim \int_{B_{c R}^{\mathbb{R}}}\left|\nabla_{\mathbb{H}} f(g)\right|^{2} d \mu(g),
$$

where $c>0$ is a universal constant.

\section{References}

[1] P. Assouad, Plongements lipschitziens dans $\mathbf{R}^{n}$. Bull. Soc. Math. France 111 (1983), 429-448. Zbl 0597.54015 MR 763553 
[2] K. Ball, E. A. Carlen, and E. H. Lieb, Sharp uniform convexity and smoothness inequalities for trace norms. Invent. Math. 115 (1994), 463-482. Zbl 0803.47037 MR 1262940

[3] Y. Benyamini and J. Lindenstrauss, Geometric nonlinear functional analysis. Vol. 1, Amer. Math. Soc. Colloq. Publ. 48, Amer. Math. Soc., Providence, RI, 2000. Zbl 0946.46002 MR 1727673

[4] A. Brunel and L. Sucheston, Sur quelques conditions équivalentes à la super-réflexivité dans les espaces de Banach. C. R. Acad. Sci. Paris Sér. A 275 (1972), 993-994. Zbl 0245.46016 MR 0318843

[5] D. Burago, Y. Burago, and S. Ivanov, A course in metric geometry. Grad. Stud. Math. 33, Amer. Math. Soc., Providence, RI, 2001. Zbl 0981.51016 MR 1835418

[6] J. Cheeger and B. Kleiner, On the differentiability of Lipschitz maps from metric measure spaces to Banach spaces. In Inspired by S. S. Chern, Nankai Tracts Math. 11, World Sci. Publ., Singapore 2006, 129-152. Zbl 1139.58004 MR 2313333

[7] J. Cheeger and B. Kleiner, Differentiating maps into $L^{1}$, and the geometry of BV functions. Ann. of Math. (2) 171 (2010), 1347-1385. Zbl 1194.22009 MR 2630066

[8] J. Cheeger, B. Kleiner, and A. Naor, Compression bounds for Lipschitz maps from the Heisenberg group to $L_{1}$. Acta Math. 207 (2011), 291-373. Zbl 1247.46020 MR 2892612

[9] J. Cheeger, B. Kleiner, and A. Naor, A $(\log n)^{\Omega(1)}$ integrality gap for the sparsest cut SDP. In 2009 50th Annual IEEE Symposium on Foundations of Computer Science (FOCS 2009), IEEE Computer Soc., Los Alamitos, CA 2009, 555-564. MR 2648435

[10] Y. de Cornulier, R. Tessera, and A. Valette, Isometric group actions on Hilbert spaces: growth of cocycles. Geom. Funct. Anal. 17 (2007), 770-792. Zbl 1129.22004 MR 2346274

[11] N. Dunford and J. T. Schwartz, Linear operators. Part I. Wiley Classics Library, John Wiley \& Sons, New York 1988. Zbl 0635.47001 MR 1009162

[12] G. B. Folland, Harmonic analysis in phase space. Ann. of Math. Stud. 122, Princeton University Press, Princeton, NJ, 1989. Zbl 0682.43001 MR 983366

[13] M. Gromov, Geometric group theory (Sussex, 1991), vol. 2: Asymptotic invariants of infinite groups. London Math. Soc. Lecture Note Ser. 182, Cambridge University Press, Cambridge 1993. Zbl 0841.20039 MR 1253544

[14] A. Guichardet, Sur la cohomologie des groupes topologiques. II. Bull. Sci. Math. (2) 96 (1972), 305-332. Zbl 0243.57024 MR 0340464

[15] A. Gupta, R. Krauthgamer, and J. R. Lee, Bounded geometries, fractals, and low-distortion embeddings. In 44th Symposium on Foundations of Computer Science (FOCS 2003), IEEE Computer Society, Washington, DC, 2003, 534-543.

[16] D. Jerison, The Poincaré inequality for vector fields satisfying Hörmander's condition. Duke Math. J. 53 (1986), 503-523. Zbl 0614.35066 MR 850547

[17] B. Kleiner, A new proof of Gromov's theorem on groups of polynomial growth. J. Amer. Math. Soc. 23 (2010), 815-829. Zbl 1246.20038 MR 2629989

[18] J. R. Lee and A. Naor, $L_{p}$ metrics on the Heisenberg group and the Goemans-Linial conjecture. In 47th Annual IEEE Symposium on Foundations of Computer Science (FOCS 2006), IEEE Computer Society, Washington, DC, 2006, 99-108. 
[19] M. Mendel and A. Naor, Towards a calculus for non-linear spectral gaps. In SODA '10: Proceedings of the Twenty-First Annual ACM-SIAM Symposium on Discrete Algorithms, SIAM, Philadelphia, PA, 2010, 236-255.

[20] A. Naor, $L_{1}$ embeddings of the Heisenberg group and fast estimation of graph isoperimetry. In Proc. Internat. Congr. Mathematicians, Vol. III, Hindustan Book Agency, New Delhi 2010, 1549-1575. Zbl 1232.46021 MR 2827855

[21] A. Naor and Y. Peres, $L_{p}$ compression, traveling salesmen, and stable walks. Duke Math. J. 157 (2011), 53-108. Zbl 05883139 MR 2783928

[22] P. Pansu, Métriques de Carnot-Carathéodory et quasiisométries des espaces symétriques de rang un. Ann. of Math. (2) 129 (1989), 1-60. Zbl 0678.53042 MR 979599

[23] G. Pisier, Martingales with values in uniformly convex spaces. Israel J. Math. 20 (1975), 326-350. Zbl 0344.46030 MR 0394135

[24] S. Rao, Small distortion and volume preserving embeddings for planar and Euclidean metrics. In Proceedings of the Fifteenth Annual Symposium on Computational Geometry (Miami Beach, FL, 1999), ACM, New York 1999, 300-306. MR 1802217

[25] S. Semmes, On the nonexistence of bilipschitz parameterizations and geometric problems about $A_{\infty}$-weights. Rev. Mat. Iberoamericana 12 (1996), 337-410. Zbl 0858.46017 MR 1402671

[26] R. Tessera, Quantitative property A, Poincaré inequalities, $L^{p}$-compression and $L^{p}$-distortion for metric measure spaces. Geom. Dedicata 136 (2008), 203-220. Zbl 1162.46043 MR 2443353

[27] R. Tessera, Asymptotic isoperimetry on groups and uniform embeddings into Banach spaces. Comment. Math. Helv. 86 (2011), 499-535. Zbl 05919456 MR 2803851

Received July 23, 2010; revised March 2, 2012

T. Austin, Courant Institute of Mathematical Sciences, New York University, 251 Mercer Street, New York, NY 10012, U.S.A.

E-mail: tim@cims.nyu.edu

A. Naor, Courant Institute of Mathematical Sciences, New York University, 251 Mercer Street, New York, NY 10012, U.S.A.

E-mail: naor@cims.nyu.edu

R. Tessera, École normale supérieure de Lyon, Unité de Mathématiques Pures et Appliquées, UMR CNRS 5669, 46 allée d'Italie, 69364 LYON Cedex 07, France

E-mail: tessera@phare.normalesup.org 